\title{
The gut microbiota and inflammatory bowel disease
}

\author{
Katsuyoshi Matsuoka $\cdot$ Takanori Kanai
}

Received: 4 August 2014 / Accepted: 2 October 2014 /Published online: 25 November 2014

(C) The Author(s) 2014. This article is published with open access at Springerlink.com

\begin{abstract}
Inflammatory bowel disease (IBD) is a chronic and relapsing inflammatory disorder of the gut. Although the precise cause of IBD remains unknown, the most accepted hypothesis of IBD pathogenesis to date is that an aberrant immune response against the gut microbiota is triggered by environmental factors in a genetically susceptible host. The advancement of next-generation sequencing technology has enabled identification of various alterations of the gut microbiota composition in IBD. While some results related to dysbiosis in IBD are different between studies owing to variations of sample type, method of investigation, patient profiles, and medication, the most consistent observation in IBD is reduced bacterial diversity, a decrease of Firmicutes, and an increase of Proteobacteria. It has not yet been established how dysbiosis contributes to intestinal inflammation. Many of the known IBD susceptibility genes are associated with recognition and processing of bacteria, which is consistent with a role of the gut microbiota in the pathogenesis of IBD. A number of trials have shown that therapies correcting dysbiosis, including fecal microbiota transplantation and probiotics, are promising in IBD.
\end{abstract}

Keywords Inflammatory bowel disease - Ulcerative colitis · Crohn's disease · Dysbiosis

This article is a contribution to the Special Issue on Microbiome, Immunity and Inflammation - Guest Editor: Hiroshi Ohno

K. Matsuoka $\cdot$ T. Kanai $(\bowtie)$

Division of Gastroenterology and Hepatology, Department of

Internal Medicine, Keio University School of Medicine, 35

Shinano-machi, Shinjuku, Tokyo 160-8582, Japan

e-mail: takagast@z2.keio.jp

\section{Introduction}

Inflammatory bowel disease (IBD) is a disorder characterized by chronic and relapsing intestinal inflammation and is mainly defined as either ulcerative colitis (UC) or Crohn's disease (CD). Although the cause of IBD remains unknown, genetic background is considered to be involved in the pathophysiology of IBD because a number of disease susceptibility genes have been identified. The rapid increase in the incidence of IBD, however, cannot be explained by genetic factors alone, and environmental factors must also be essential to its development.

The involvement of the gut microbiota in the pathophysiology of IBD has recently been highlighted. Several lines of evidence suggest an essential role of the gut microbiota in intestinal inflammation. (1) In murine models of IBD such as IL-10-deficient mice and the $\mathrm{CD} 45 \mathrm{Rb}^{\text {high }}$ transfer model, where transferred naïve helper $\mathrm{T}$ cells cause microbiota-dependent intestinal inflammation in immune-deficient recipients such as $\mathrm{Rag} 2^{-/-}$ mice, germ-free animals do not develop colitis. (2) Diversion of the fecal stream ameliorates intestinal inflammation in CD [1]. (3) Antibiotics are, to a certain degree, effective for the treatment of IBD [2]. (4) Antibiotics such as metronidazole and ciprofloxacin are also effective for anal lesions and prevention of postoperative recurrence in CD [3]. (5) Many of the reported IBD susceptibility genes are associated with recognition and processing of microbes [4].

Given these observations, the most accepted hypothesis of IBD pathogenesis to date is that an aberrant immune response against the gut microbiota is triggered by environmental factors in a genetically susceptible host. In this review, we will discuss abnormalities of the gut microbiota observed in IBD, their contribution to the pathogenesis of IBD, and related therapeutic applications. 


\section{Alteration of the gut microbiota in IBD}

Dysbiosis

The host provides a nutrient-rich environment and residence for the gut bacteria, and in turn, they contribute to the host by producing short-chain fatty acids and essential vitamins. This mutual relationship between the host and the gut bacteria is called symbiosis. Recent advancement of next-generation sequencing techniques has enabled culture-independent analysis of the gut microbiota, revealing that an altered balance of the gut microbiota constituents, rather than specific pathogens, is involved in the pathophysiology of several diseases. This shift in the balance of the gut microbiota is referred to as dysbiosis.

More than $90 \%$ of the human gut microbiota is composed of four major phyla. The Firmicutes (49-76\%) and Bacteroidetes (16-23\%) phyla dominate, followed to a much less extent by the Proteobacteria and Actinobacteria phyla [5, 6]. The Firmicutes phylum is mainly composed of the Clostridium XIV and IV groups.

Various alterations of the gut microbiota have been reported in IBD patients (Table 1). Most studies have shown reduced diversity of the gut microbiota in IBD patients [6-9]. The most consistent observations of altered composition of the gut microbiota in IBD patients are a reduction in Firmicutes and an increase in Proteobacteria [6, 7, 10-12]. The reduced diversity of the gut microbiota observed in IBD patients is largely due to a decline in the diversity of Firmicutes. Among Firmicutes, a decrease in the Clostridium leptum groups, especially Faecalibacterium prausnitzii, has been reported in many studies [7, 13, 14]. Results related to Enterobacteriaceae, Bacteroides, Bifidobacteria species, Lactobacillus species, and Escherichia coli are not consistent among studies $[15,16]$. Various factors may explain the between-study discrepancies: (1) sample source (biopsy or stool), (2) sampling location (inflammatory or noninflammatory sites), (3) disease activity (active or quiescent), (4) medication, (5) diet, (6) age, (7) smoking, and (8) methods used to analyze the microbiota.

While the gut microbiota in healthy subjects shows little temporal change, the gut microbiota in IBD patients is unstable. The composition of the gut microbiota differs between active and quiescent stages. Furthermore, a study that longitudinally examined the gut microbiota in IBD patients for a year demonstrated that the gut microbiota was unstable even in UC patients in remission [17]. Before relapse of UC, normal anaerobic bacteria such as Bacteroides, Escherichia, Eubacterium, Lactobacillus, and Ruminococcus are decreased and the diversity of the gut microbiota is also reduced [18]. In CD patients, dysbiosis is observed even in patients with remission. Medication also affects the composition of the gut microbiota. Mesalazine, for example, reduces the total bacterial number to almost half [19]. Bowel rest, which is a treatment option in $\mathrm{CD}$, changes the composition of the gut microbiota. Antibiotics dramatically amplify the dysbiosis of CD [20].

The distribution of the gut microbiota should be taken into account when interpreting dysbiosis. For example, the composition of the microbiota is significantly different between fecal and mucosal samples [5, 21]. Mucosal samples are reported to be superior in order to detect dysbiosis [20]. The mucosa-associated microbiota is increased in IBD compared with healthy subjects $[22,23]$. It is tempting to speculate that the mucosa-associated microbiota is physiologically more important in IBD than luminal microbiota because of the close contact of mucosa-associated bacteria with the intestinal surface. It has also been reported that the gut microbiota is different in the same individual between inflammatory and noninflammatory sites [24]. The dysbiosis observed in noninflammatory sites may be more representative of a causative composition because the dysbiosis observed in inflammatory sites may be affected by inflammation.

It remains controversial whether dysbiosis is a cause or consequence of intestinal inflammation in IBD. Comparison of the gut microbiota composition of IBD patients with that of their unaffected relatives, who are likely to share genetic and environmental background, is useful to provide evidence relevant to this fundamental question. Compositional change of the gut microbiota was not consistent between UC patients and their unaffected twins [25]. In contrast, a decrease in F. prausnitzii was reported to be observed in both UC patients and their first-grade relatives [26]. Unaffected relatives of CD patients also had dysbiosis, although it was different from the dysbiosis observed in CD patients [27]. Furthermore, it was reported that the genetic status of NOD2 and $A T G 18 L$ genes, which are two major CD susceptibility genes, was associated with alteration of the gut microbiota [28]. These results suggest that dysbiosis is caused by genetic and environmental factors, rather than being a consequence of inflammation.

There have been attempts to utilize dysbiosis as a diagnostic tool or biomarker [9]. To date, there are no microbial constituents specific to UC or CD, because interindividual variations are much larger than inter-disease differences [6]. Several studies have suggested the possibility of using the gut microbiota as a biomarker. Firmicutes, for example, was increased in UC patients who responded to mesalazine [19]. The relapse rate was lower in postoperative $C D$ patients who had a similar composition of gut microbiota to healthy controls than in those with dysbiosis [29]. In the largest CD microbiota cohort so far, comparing 447 newly diagnosed pediatric CD patients with 221 healthy controls, Gevers et al. proposed a "dysbiosis index," which was shown to be associated with clinical disease severity assessed using the Pediatric Crohn's Disease Index [20]. They also reported that profiles of the gut microbiota were able to be utilized as a diagnostic marker of $\mathrm{CD}$ and were also useful to predict the severity at 6 months. 
Table 1 Metagenomic analysis of the gut microbiota in inflammatory bowel disease

\begin{tabular}{|c|c|c|c|c|c|c|c|c|c|c|}
\hline \multirow{2}{*}{$\begin{array}{l}\text { Sample } \\
\text { source }\end{array}$} & \multicolumn{3}{|c|}{ Sample no. } & \multirow[t]{2}{*}{ Diversity } & \multirow{2}{*}{$\begin{array}{l}\text { Bacterial } \\
\text { no. }\end{array}$} & \multirow[t]{2}{*}{ Firmicutes } & \multirow[t]{2}{*}{ Bacteroidetes } & \multirow[t]{2}{*}{ Actinobacteria } & \multirow[t]{2}{*}{ Proteobacteria } & \multirow[t]{2}{*}{ Ref. } \\
\hline & $\mathrm{CD}$ & $\mathrm{UC}$ & $\mathrm{HC}$ & & & & & & & \\
\hline Stool & 6 & - & 6 & $\downarrow$ in $\mathrm{CD}$ & & $\downarrow$ in $\mathrm{CD}$ & $\rightarrow$ in IBD & & & {$[7]$} \\
\hline Biopsy & 6 & 5 & 5 & & & $\downarrow$ in $C D$ & $\uparrow$ in $\mathrm{CD}$ & $\uparrow$ in IBD & $\uparrow$ in $\mathrm{CD}$ & [10] \\
\hline $\begin{array}{r}\text { Surgical } \\
\text { tissue }\end{array}$ & 35 & 55 & 34 & & $\downarrow$ in IBD & $\begin{array}{l}\downarrow \text { Lachnospiraceae } \\
\uparrow \text { Bacillus }\end{array}$ & $\downarrow$ in IBD & $\begin{array}{l}\uparrow \text { Bifidobacteriaceae } \\
\text { in cCD } \\
\uparrow \text { Coriobacteriaceae } \\
\text { in cCD }\end{array}$ & $\uparrow$ in IBD & {$[6]$} \\
\hline Stool & 29 & 16 & 35 & $\downarrow$ in CD & $\downarrow$ in CD & $\begin{array}{l}\uparrow \text { in } \mathrm{cCD}, \downarrow \text { in iCD } \\
\uparrow \text { Ruminococcaceae in cCD } \\
\downarrow \text { Ruminococcaceae in iCD }\end{array}$ & & & $\begin{array}{l}\uparrow \text { Enterobacteriaceae } \\
\text { in CD }\end{array}$ & {$[8]$} \\
\hline Biopsy & 6 & 6 & 5 & $\downarrow$ in IBD & $\downarrow$ in $\mathrm{CD}$ & $\begin{array}{l}\downarrow \text { in IBD } \\
\rightarrow F \text {. prausnitzii in IBD }\end{array}$ & $\uparrow$ in IBD & & $\begin{array}{l}\uparrow \text { Enterobacteriaceae } \\
\text { in CD }\end{array}$ & [24] \\
\hline $\begin{array}{l}\text { Biopsy } \\
\text { Stool }\end{array}$ & 121 & 75 & 27 & & & $\downarrow$ in $\mathrm{CD}$ & & $\uparrow$ in IBD & $\begin{array}{l}\uparrow \text { Enterobacteriaceae } \\
\text { in CD }\end{array}$ & [49] \\
\hline $\begin{array}{l}\text { Endoscopic } \\
\text { lavage }\end{array}$ & 16 & 16 & 32 & $\downarrow$ in IBD & & $\begin{array}{l}\downarrow \text { in IBD } \\
\downarrow \text { F. prausnitzii in IBD }\end{array}$ & & & $\uparrow$ in IBD & [9] \\
\hline Stool & 21 & 34 & 21 & & $\rightarrow$ & $\begin{array}{c}\downarrow \text { C. coccoides and } \\
\text { C. leptum in IBD } \\
\uparrow \text { Lactobacillus in CD } \\
\downarrow \text { F. prausnitzii in IBD }\end{array}$ & & $\begin{array}{l}\uparrow \text { Bifidobacterium in } \\
\text { UC }\end{array}$ & $\uparrow E$. coli in $\mathrm{CD}$ & [14] \\
\hline Biopsy & 29 & 15 & 21 & & $\downarrow$ in IBD & $\begin{array}{l}\downarrow \text { C. coccoides in CD } \\
\downarrow \text { C. leptum in IBD } \\
\uparrow \text { Lactobacillus in CD } \\
\downarrow \text { F. prausnitzii in IBD }\end{array}$ & $\uparrow$ in IBD & $\begin{array}{l}\downarrow \text { Bifidobacteriaceae } \\
\text { in } \mathrm{CD}\end{array}$ & $\uparrow E$. coli in IBD & [14] \\
\hline $\begin{array}{l}\text { Biopsy and } \\
\text { stool }\end{array}$ & 447 & - & 221 & $\downarrow$ in CD & & $\downarrow$ Clostridiales in CD & $\downarrow$ in $\mathrm{CD}$ & & $\begin{array}{l}\uparrow \text { Enterobacteriaceae } \\
\text { in CD }\end{array}$ & [20] \\
\hline
\end{tabular}

$C D$ Crohn's disease, $U C$ ulcerative colitis, $I B D$ inflammatory bowel disease, $i C D$ ileal $\mathrm{CD}, c C D$ colonic $\mathrm{CD}$

This report encourages further efforts to use gut microbial profiles as a diagnostic tool or biomarker for disease activity, prognosis, and response to treatment.

Specific bacteria associated with IBD

There have been no specific pathogens yet identified that fulfill Koch's postulates. There are, however, several specific bacteria that are associated with IBD. Mycobacterium avium subspecies paratuberculosis (MAP) causes chronic granulomatous ileitis (Johne's disease) in cattle and sheep, which shares some pathological features with CD. In addition, because MAP has been found in commercial milk, it is suspected as a causative pathogen of CD. MAP was detected in CD patients using mucosal PCR, and the positive rates of serum anti-MAP antibody were higher in CD patients than in healthy controls or UC patients [30]. However, a clinical trial of a 2year administration of antituberculosis drugs to CD patients showed no efficacy [31]. Adhesive-invasive E. coli (AIEC), which can adhere to and invade the intestinal epithelial cells, colonize the ileal mucosa of CD [32]. AIEC also replicate in macrophages and stimulate $\mathrm{TNF} \alpha$ production from macrophages. It was observed that Fusobacterium varium attaches to inflamed regions in UC and invades the mucosa at ulcers [33]. Serum titers for anti-F. varium antibodies were higher in UC patients compared with healthy controls [34]. $F$ varium produces butyrate, and rectal administration of butyrate has been shown to cause mucosal damage in mice [35]. The pathological consequence of butyrate production by $F$. varium, however, needs to be examined more extensively because butyrate has diverse effects on the intestinal homeostasis including Treg induction in the gut and energy supply to the intestinal epithelial cells. The combination therapy of amoxicillin, tetracycline, and metronidazole (to which $F$ varium is sensitive) for 2 weeks showed efficacy in active UC patients, suggesting the possible pathogenic role of F. varium [36].

The role of the gut microbiota in IBD revealed by susceptibility genes

The association of IBD susceptibility genes with bacteria has recently been highlighted. The development of the genomewide association study has contributed greatly to the 
identification of more than 160 IBD susceptibility genes to date [4]. The physiological functions of these genes are categorized into several groups relating to (1) acquired immunity (IL23R, IL12B, JAK2, STAT3), (2) bacterial recognition and processing (NOD2/CARD15), (3) autophagy (ATG16L, IRGM, ATG5), and (4) mucosal barrier (ECM1, CDH1, $L A M B 1$ ) [37]. Many of the CD susceptibility genes are associated with bacterial recognition and processing, and many of the UC susceptibility genes are related to mucosal barrier function, suggesting that impaired handling of bacteria or disruption of the mucosal barrier function leads to breakdown of tolerance against the commensal bacterial in the gut in $\mathrm{CD}$ and $U C$, respectively.

NOD2/CARD15 was the first reported CD susceptible gene and shows the strongest association with $\mathrm{CD}$. The function of the NOD2 protein has been extensively studied. NOD2 is an intracellular receptor for muramyl dipeptide (MDP), a component of the cell wall of gram-positive bacteria, and is expressed in intestinal epithelial cells and monocytes/macrophages. While Nod2-deficient mice do not develop spontaneous colitis, the bacterial load in the gut is increased in these mice. CD patients with NOD2 mutations demonstrate diminished production of antimicrobial peptides (AMPs) from Paneth cells [38] as well as reduced production of the antiinflammatory cytokine IL-10 from peripheral mononuclear cells [39]. NOD2 stimulation with MDP induces autophagy [40], which regulates replication of intracellular bacteria and is also involved in bacterial antigen presentation in the infected cells.

Several autophagy-related genes have also been reported as $\mathrm{CD}$ susceptibility genes. Autophagy is an intracellular process that is involved in degradation and recycling of proteins when cells are in starvation. Autophagy is also involved in the handling of intracellular pathogens. $A T G 16 L$ is a susceptibility gene for $\mathrm{CD}$ and is essential for autophagosome formation. Interestingly, it has been shown that NOD1 and NOD2 sense bacterial invasion into the cell and recruit ATG16L to the site of bacterial entry, which triggers autophagy. The intracellular bacteria are then processed through autophagy [40]. This close association between NOD2 and ATG16L suggests the importance of this pathway in the pathophysiology of CD.

Studies on IBD susceptibility genes have revealed the essential role of Paneth cells in CD. Paneth cells reside at the bottom of the intestinal crypts and produce AMPs. The important role of Paneth cells in the regulation of the gut microbiota and the intestinal immune system is shown by the observation that genetically engineered mice overexpressing $\alpha$-defensin, one of the AMPs, in intestinal epithelial cells had a reduced number of segmented filamentous bacteria (SFB) in the gastrointestinal tract, resulting in impaired Th17 development in the gut [41]. Abnormalities in the size, number, and distribution of granules in Paneth cells, which contain AMPs, have been observed in CD. These morphological abnormalities were reported to be more frequent in CD patients with NOD2 or $A T G 16 L$ mutations [42]. Mice harboring the same Atg16l mutation as CD patients develop similar morphological abnormalities of Paneth cells after murine Norovirus infection and become susceptible to dextran sodium sulfate (DSS)-induced colitis [43]. These results provide a good example of the complex interaction between a genetic factor and an environmental factor in the development of intestinal inflammation. Mice deficient in $X b p 1$, which is an essential molecule for endoplasmic reticulum stress and is a CD susceptibility gene, also have impaired autophagy induction in Paneth cells and develop spontaneous ileitis [44]. These results suggest that autophagy in Paneth cells is critical for maintaining gut homeostasis, probably through regulation of the gut microbiota by AMP production. Impaired Paneth cell function may be an essential element in the development and perpetuation of intestinal inflammation in $\mathrm{CD}$.

How does dysbiosis lead to intestinal inflammation?

It is well known that different commensal bacteria induce distinct types of colitis in IL-10-deficient mice. A monoassociation study, in which a single strain of bacteria was inoculated into germ-free IL-10-deficient mice, demonstrated that $E$. coli induced cecal inflammation, Enterococcus faecalis induced distal colitis, and Pseudomonas fluorescens did not cause colitis [45]. It was also reported that the presence of Helicobacter hepaticus, a species of commensal bacteria, exacerbated colitis in IL-10-deficient mice. These results show that alteration of the composition of the gut microbiota can cause distinct intestinal immune responses even in a host with the same genetic background, suggesting that dysbiosis can modulate the immune response in the gut.

Garrett et al. [46] reported that mice deficient in both Tbx21/T-bet, which is an essential transcription factor for Th1 differentiation, and Rag, which is indispensable for the acquired immune system, developed spontaneous UC-like colitis, which was ameliorated by the administration of antibiotics. Importantly, wild-type mice co-housed with colitic $T$ bet/Rag double knockout mice also developed similar colitis, suggesting that a dysbiotic gut microbiota is communicable and can cause intestinal inflammation without genetic manipulation.

Functional changes in the gut microbiota resulting from dysbiosis may be involved in the pathophysiology of IBD. The number of genes harbored in the gut microbiota is 100 times greater than that in the human genome [5, 47, 48]. Metabolites of the gut microbiota contribute to epithelial cell function, energy balance, and the immune system of the host. A metagenomic analysis of the gut microbiota showed a decrease in genes responsible for carbohydrate and amino acid metabolism and an increase in those in the oxidative 
stress pathway, in IBD patients [49], raising the possibility that oxidative stress from the gut microbiota causes intestinal inflammation in IBD patients. A specific metabolite of the gut microbiota is also likely to be involved in the pathophysiology of IBD. The gut microbiota metabolizes nonabsorptive dietary fiber and produces short-chain fatty acids such as butyrate and propionate. Commensal bacteria-derived butyrate induces the differentiation of colonic regulatory $\mathrm{T}$ cells in mice [50]. Butyrate is also an important energy source for intestinal epithelial cells and increases production of mucin and AMPs [13]. The concentrations of butyrate in feces have consistently been shown to be decreased in IBD patients. Consistently, F prausnitzii, a species of butyrate-producing bacteria, has also been observed to be decreased in IBD [16]. It is possible that the decreased level of butyrate in the gut contributes to inducing intestinal inflammation. Another example of a functional alteration of the gut microbiota in IBD is the increase of sulfate-reducing bacteria (SRB) in UC [51]. SRB produce hydrogen sulfide, which is toxic to the intestinal epithelial cells and can cause mucosal inflammation.

Recent studies have revealed that specific bacteria control the intestinal immune system. SFB, for example, induce Th17 cells in the murine intestine [52]. Although the human counterpart of SFB has not yet been identified, SFB-like organisms were observed in six out of six surgical specimens from UC patients [53]. These SFB-like organisms were not observed in surgical specimens from CD patients. In non-IBD controls, SFB-like organisms were observed in three out of six specimens, with a much lower density compared with UC. These are interesting observations because Th17 cells were reported to be increased in IBD. The physiological role of these SFBlike organisms requires further investigation.

The number of bacteria in the mucus layer is increased in IBD [22], suggesting impaired mucosal barrier function. This is consistent with the fact that many of the UC susceptibility genes are related to mucosal barrier function. Furthermore, bacteria that can degrade mucins in the mucus layer and utilize it as an energy source, for example Ruminococcus gnavus and Ruminococcus torques, are increased in IBD. These bacteria help other bacteria reside in the mucus layer by providing degraded mucins as nutrients.

\section{IBD therapies targeting the gut microbiota}

Fecal microbiota transplantation

Fecal microbiota transplantation (FMT) is a treatment to restore abnormal microbial composition of the gut by introducing fecal microbiota obtained from a healthy donor into a diseased individual. The results of a randomized study to compare FMT with antibiotics for recurrent Clostridium difficile infection were striking [54]. Resolution of C. difficileassociated diarrhea was observed in 13 of 16 patients $(81 \%)$ in the FMT group, compared with four out of 13 patients $(31 \%)$ in the antibiotics group.

FMT has been highlighted as a treatment to correct dysbiosis in IBD. The first implementation of FMT in UC was reported in 1989 [55]. One of the authors of the paper received FMT for his continuously active $\mathrm{UC}$, resulting in drug-free remission. A recent systematic review identified 18 UC patients without $C$. difficile infection who were treated with FMT [56]. Thirteen out of the 18 patients experienced disease resolution; however, selection bias should be considered because all of the cases were from case reports or small case series.

Two prospective studies of FMT for adult UC patients were recently published $[57,58]$. Both of these studies longitudinally analyzed the change of bacterial composition in FMT recipients. Unexpectedly, none of the combined 11 patients in the two studies achieved clinical remission after FMT. In contrast, a significant change in the gut microbiota composition was observed in most of the patients. One paper reported that the successful colonization of donor microbiota was correlated with clinical improvement in one patient, but the other study did not confirm this finding. Both of the papers reported that the alteration of gut microbiota was temporary in most patients, suggesting the necessity of periodically repeated transplantation to maintain the altered gut microbiota.

A phase I trial of FMT for 10 pediatric UC patients with mild-to-moderate activity has recently been completed, reporting no serious adverse events and a high rate of clinical response (79\%) within 1 week [59]. This result is in contrast to the above-mentioned studies. One possible reason to explain this discrepancy is that a certain population of $\mathrm{UC}$ patients, but not all, may benefit from FMT. Interestingly, Angelberger et al. identified phylotypes that are indicative of disease severity and FMT success, specifically overpresentation of Enterobacteriaceae and under-repression of Lachnospiraceae [57]. This is an important factor in selecting a subgroup of UC patients that may be responsive to FMT.

\section{Probiotics}

Probiotics are preparations utilizing live bacteria that can be beneficial to human health. Several reports have shown the efficacy of various probiotic bacteria for IBD (Table 2). Efficacy of probiotics was studied more extensively in UC than CD. VSL\#3 is a freeze-dried preparation containing eight different lactic acid bacteria (Lactobacillus acidophilus, L. bulgaricus, L casei, L. plantarum, Streptococcus thermophilus, Bifidobacterium breve, B. infantis, and B. longum). Two double-blinded placebo-controlled trials 
Table 2 Randomized controlled trials of probiotics for inflammatory bowel disease

\begin{tabular}{|c|c|c|c|c|c|c|}
\hline Probiotics & Disease & Endpoint & Groups and subject no. & Duration & Conclusion & Ref. \\
\hline \multirow[t]{5}{*}{ VSL\#3 } & $\mathrm{UC}$ & Induction & $\begin{array}{l}\text { Conventional therapy+VSL\#3, } 77 \\
\text { Conventional therapy+ placebo: } 70\end{array}$ & 12 weeks & Effective & {$[63]$} \\
\hline & $\mathrm{UC}$ & Induction & $\begin{array}{l}\text { Conventional therapy+VSL\#3, } 71 \\
\text { Conventional therapy+placebo, } 73\end{array}$ & 8 weeks & Effective & {$[62]$} \\
\hline & UC & $\begin{array}{l}\text { Induction } \\
\text { Maintenance }\end{array}$ & $\begin{array}{l}\text { Steroid/mesalazine+VSL\#3, } 14 \\
\text { Steroid/mesalazine+ placebo, } 15\end{array}$ & 1 year & Effective & {$[64]$} \\
\hline & Pouchitis & Maintenance & $\begin{array}{l}\text { VSL } \# 3,20 \\
\text { Placebo, } 20\end{array}$ & 9 months & Effective & {$[60]$} \\
\hline & Pouchitis & Maintenance & $\begin{array}{l}\text { VSL\#3, } 20 \\
\text { Placebo, } 16\end{array}$ & 12 months & Effective & {$[61]$} \\
\hline \multirow[t]{3}{*}{ Nissle 1917} & $\mathrm{UC}$ & Induction & $\begin{array}{l}\text { Steroid }+ \text { mesalazine, } 57 \\
\text { Steroid }+ \text { Nissle } 1917,59\end{array}$ & 12 weeks & $\begin{array}{l}\text { Equivalent to } \\
\text { mesalazine }\end{array}$ & {$[66]$} \\
\hline & $\mathrm{UC}$ & Maintenance & $\begin{array}{l}\text { Nissle } 1917,162 \\
\text { Mesalazine, } 165\end{array}$ & 12 months & $\begin{array}{l}\text { Equivalent to } \\
\text { mesalazine }\end{array}$ & {$[67]$} \\
\hline & $\mathrm{UC}$ & Maintenance & $\begin{array}{l}\text { Mesalazine, } 50 \\
\text { Nissle } 1917,53\end{array}$ & 12 weeks & $\begin{array}{l}\text { Equivalent to } \\
\text { mesalazine }\end{array}$ & {$[68]$} \\
\hline \multirow[t]{2}{*}{ Lactobacillus GG } & $\mathrm{CD}$ & Maintenance & $\begin{array}{l}\text { Conventional therapy }+ \text { Lactobacillus } \mathrm{GG} \text {, } \\
39 \\
\text { Conventional therapy }+ \text { placebo, } 36\end{array}$ & $\sim 2$ years & Not effective & [71] \\
\hline & $\mathrm{UC}$ & Maintenance & $\begin{array}{l}\text { Lactobacillus } \mathrm{GG}, 65 \\
\text { Lactobacillus } \mathrm{GG}+\text { mesalazine, } 62 \\
\text { Mesalazine, } 60\end{array}$ & 12 months & $\begin{array}{l}\text { Equivalent to } \\
\text { mesalazine }\end{array}$ & {$[70]$} \\
\hline \multirow[t]{2}{*}{$\begin{array}{l}\text { Bifidobacteria-fermented } \\
\text { milk (BFM) }\end{array}$} & $\mathrm{UC}$ & Induction & $\begin{array}{l}\text { Conventional therapy+BFM, } 10 \\
\text { Conventional therapy+placebo, } 10\end{array}$ & 12 weeks & Effective & {$[74]$} \\
\hline & $\mathrm{UC}$ & Maintenance & $\begin{array}{l}\text { Conventional therapy+BFM, } 11 \\
\text { Conventional therapy, } 10\end{array}$ & 12 months & Effective & {$[75]$} \\
\hline $\begin{array}{l}\text { Bifidobacterium longum/Synergy } \\
1\end{array}$ & $\mathrm{UC}$ & Induction & $\begin{array}{l}\text { Bifidobacterium longum/Synergy 1, } 9 \\
\text { Placebo, } 9\end{array}$ & 1 month & Effective & {$[73]$} \\
\hline
\end{tabular}

have shown the efficacy of VSL\#3 in the prevention of recurrence in patients with chronic relapsing pouchitis [60, 61]. Two randomized controlled clinical trials showed that addition of VSL\#3 to conventional therapy was more efficacious in remission induction in active UC patients [62, 63]. Another randomized controlled trial compared the efficacy of addition of VSL\#3 to mesalazine and steroids in 29 newly diagnosed pediatric UC patients with addition of a placebo [64]. The remission rates at 4 weeks were significantly higher in the VSL\#3 group $(92.8 \%(13 / 14))$ than in the placebo group $(36.4 \%(4 / 15))$. Furthermore, the recurrence rates at 1 year were $36.4 \%(3 / 14)$ and $73.3 \%(11 / 15)$ in the VSL\#3 and placebo groups, respectively. These results show that VSL\#3 is effective for induction of remission as well as its maintenance in UC patients. The mechanism of the antiinflammatory effect of VSL\#3 is not yet fully understood but an increase in regulatory $\mathrm{T}$ cells in the gut and upregulation of mucosal alkaline sphingomyelinase have been reported [65].

Nissle 1917, a nonpathogenic E. coli strain, showed efficacy in maintenance of remission in UC equivalent to mesalazine
[66-68]. Nissle 1917 inhibits IL-8 production stimulated by $\mathrm{TNF} \alpha$ from the epithelial cells [69]. Lactobacillus $G G$ can be effective for maintenance of remission in UC patients [70], but not in CD patients [71, 72]. A pilot study demonstrated that Bifidobacterium longum/Synergy 1 improved intestinal inflammation in active UC patients [73]. Bificobacteria-fermented milk also demonstrated efficacy in induction and maintenance of remission in UC patients [74, 75].

We reported that Clostridium butyricum (CB), which is used as a probiotic for patients with functional gastrointestinal disorders in a clinical setting, suppresses intestinal inflammation in murine IBD models [76]. CB potently induced the antiinflammatory cytokine IL-10 from colonic mucosal macrophages. This IL-10 production was dependent on the Toll-like receptor 2/MyD88 pathway. The effect of $\mathrm{CB}$ was abolished in IL-10-deficient mice, suggesting that the anticolitic effect of $\mathrm{CB}$ was due to IL-10. Interestingly, heat-killed CB also induced IL-10 from macrophages, strongly indicating that bacterial-derived products are responsible for IL-10 induction. A clinical trial to examine the anticolitic effect of CB in IBD patients is warranted. 


\section{Closing remarks}

The gut immune system is separated from an enormous number of bacteria only by a single layer of epithelial cells. It is thus tempting to speculate that the gut microbiota is involved in the pathophysiology of IBD. The advancement of nextgeneration sequencing technology has revealed a range of alterations of the gut microbiota in IBD. However, it remains unclear whether the dysbiosis observed in IBD is a cause or a consequence of intestinal inflammation. To answer this essential question, studies to examine longitudinal changes of the microbiota in a large number of IBD patients, especially newly diagnosed patients, are necessary. Furthermore, little information is available to how dysbiosis regulates the gut immune system. Understanding the complex relationship between the gut immune system and the microbiota should lead to further elucidation of the pathogenesis of IBD and development of curative treatments. Utilizing the gut microbiota as a diagnostic tool or biomarker is also an attractive idea. To this end, a disease-specific or activity-specific core microbiome should be identified. The gut microbiota is composed not only of bacteria but also of viruses and fungi. It is important to include viruses and fungi in any investigation of the gut microbiota. Furthermore, functional analysis of the gut microbiota in IBD is warranted. Because a majority of the gut bacteria are unable to be cultured, gnotobiotic approaches will be an important tool to investigate the function of the gut microbiota. The gut microbiota represents a "gold mine" for both clinical and basic IBD research.

Acknowledgments This study was supported by Grants-in-Aid from the Japanese Ministry of Education, Culture, Sports, Science and Technology; the Health and Labour Sciences Research Grants for Research on Intractable Diseases from the Ministry of Health, Labour and Welfare of Japan; and the Keio University Medical Fund.

Conflict of interest Katsuyoshi Matsuoka received a research grant from Mitsubishi Tanabe Pharma Corporation. Takanori Kanai received a research grant from Mitsubishi Tanabe Pharma Corporation, Eisai Co., Ltd., Abbvie, JIMRO Co., Ltd., Takeda Pharmaceutical Co., Ltd., and ZERIA Co., Ltd.

Open Access This article is distributed under the terms of the Creative Commons Attribution License which permits any use, distribution, and reproduction in any medium, provided the original author(s) and the source are credited.

\section{References}

1. Rutgeerts P et al (1991) Effect of faecal stream diversion on recurrence of Crohn's disease in the neoterminal ileum. Lancet 338:771774

2. Khan KJ et al (2011) Antibiotic therapy in inflammatory bowel disease: a systematic review and meta-analysis. Am J Gastroenterol 106:661-673. doi:10.1038/ajg.2011.72
3. Ananthakrishnan AN et al (2011) Strategies for the prevention of postoperative recurrence in Crohn's disease: results of a decision analysis. Am J Gastroenterol 106:2009-2017. doi:10.1038/ajg. 2011.237

4. Jostins L et al (2012) Host-microbe interactions have shaped the genetic architecture of inflammatory bowel disease. Nature 491: 119-124. doi:10.1038/nature11582

5. Eckburg PB et al (2005) Diversity of the human intestinal microbial flora. Science 308:1635-1638. doi:10.1126/science.1110591

6. Frank DN et al (2007) Molecular-phylogenetic characterization of microbial community imbalances in human inflammatory bowel diseases. Proc Natl Acad Sci U S A 104:13780-13785. doi:10. 1073/pnas.0706625104

7. Manichanh C et al (2006) Reduced diversity of faecal microbiota in Crohn's disease revealed by a metagenomic approach. Gut 55:205211. doi:10.1136/gut.2005.073817

8. Willing BP et al (2010) A pyrosequencing study in twins shows that gastrointestinal microbial profiles vary with inflammatory bowel disease phenotypes. Gastroenterology 139(1844-1854):e1841. doi: 10.1053/j.gastro.2010.08.049

9. Tong $\mathrm{M}$ et al (2013) A modular organization of the human intestinal mucosal microbiota and its association with inflammatory bowel disease. PLoS One 8:e80702. doi:10.1371/journal.pone.0080702

10. Gophna U et al (2006) Differences between tissue-associated intestinal microfloras of patients with Crohn's disease and ulcerative colitis. J Clin Microbiol 44:4136-4141. doi:10.1128/jcm.01004-06

11. Scanlan PD et al (2006) Culture-independent analyses of temporal variation of the dominant fecal microbiota and targeted bacterial subgroups in Crohn's disease. J Clin Microbiol 44:3980-3988. doi: $10.1128 / \mathrm{jcm} .00312-06$

12. Peterson DA et al (2008) Metagenomic approaches for defining the pathogenesis of inflammatory bowel diseases. Cell Host Microbe 3: 417-427. doi:10.1016/j.chom.2008.05.001

13. Sokol $\mathrm{H}$ et al (2008) Faecalibacterium prausnitzii is an antiinflammatory commensal bacterium identified by gut microbiota analysis of Crohn disease patients. Proc Natl Acad Sci U S A 105: 16731-16736. doi:10.1073/pnas.0804812105

14. Wang $\mathrm{W}$ et al (2014) Increased proportions of Bifidobacterium and the Lactobacillus group and loss of butyrate-producing bacteria in inflammatory bowel disease. J Clin Microbiol 52:398-406. doi:10. $1128 / \mathrm{jcm} .01500-13$

15. Andoh A et al (2011) Comparison of the fecal microbiota profiles between ulcerative colitis and Crohn's disease using terminal restriction fragment length polymorphism analysis. J Gastroenterol 46:479486. doi:10.1007/s00535-010-0368-4

16. Takaishi $\mathrm{H}$ et al (2008) Imbalance in intestinal microflora constitution could be involved in the pathogenesis of inflammatory bowel disease. Int J Med Microbiol 298:463-472. doi:10.1016/j.ijmm.2007.07.016

17. Martinez $C$ et al (2008) Unstable composition of the fecal microbiota in ulcerative colitis during clinical remission. Am J Gastroenterol 103:643-648. doi:10.1111/j.1572-0241.2007.01592.x

18. Ott SJ et al (2008) Dynamics of the mucosa-associated flora in ulcerative colitis patients during remission and clinical relapse. J Clin Microbiol 46:3510-3513. doi:10.1128/jcm.01512-08

19. Andrews $\mathrm{CN}$ et al (2011) Mesalazine (5-aminosalicylic acid) alters faecal bacterial profiles, but not mucosal proteolytic activity in diarrhoea-predominant irritable bowel syndrome. Aliment Pharmacol Ther 34:374-383. doi:10.1111/j.1365-2036.2011.04732.x

20. Gevers D et al (2014) The treatment-naive microbiome in new-onset Crohn's disease. Cell Host Microbe 15:382-392. doi:10.1016/j. chom.2014.02.005

21. Lepage $\mathrm{P}$ et al (2005) Biodiversity of the mucosa-associated microbiota is stable along the distal digestive tract in healthy individuals and patients with IBD. Inflamm Bowel Dis 11:473-480

22. Swidsinski A et al (2002) Mucosal flora in inflammatory bowel disease. Gastroenterology 122:44-54 
23. Schultsz C et al (1999) The intestinal mucus layer from patients with inflammatory bowel disease harbors high numbers of bacteria compared with controls. Gastroenterology 117:1089-1097

24. Walker AW et al (2011) High-throughput clone library analysis of the mucosa-associated microbiota reveals dysbiosis and differences between inflamed and non-inflamed regions of the intestine in inflammatory bowel disease. BMC Microbiol 11:7. doi:10.1186/14712180-11-7

25. Lepage $P$ et al (2011) Twin study indicates loss of interaction between microbiota and mucosa of patients with ulcerative colitis. Gastroenterology 141:227-236. doi:10.1053/j.gastro.2011.04.011

26. Varela E et al (2013) Colonisation by Faecalibacterium prausnitzii and maintenance of clinical remission in patients with ulcerative colitis. Aliment Pharmacol Ther 38:151-161. doi:10.1111/apt.12365

27. Joossens $\mathrm{M}$ et al (2011) Dysbiosis of the faecal microbiota in patients with Crohn's disease and their unaffected relatives. Gut 60:631-637. doi:10.1136/gut.2010.223263

28. Frank DN et al (2011) Disease phenotype and genotype are associated with shifts in intestinal-associated microbiota in inflammatory bowel diseases. Inflamm Bowel Dis 17:179-184. doi:10.1002/ibd.21339

29. Dey N et al (2013) Association of gut microbiota with post-operative clinical course in Crohn's disease. BMC Gastroenterol 13:131. doi: 10.1186/1471-230x-13-131

30. Feller M et al (2007) Mycobacterium avium subspecies paratuberculosis and Crohn's disease: a systematic review and meta-analysis. Lancet Infect Dis 7:607-613. doi:10.1016/s1473-3099(07)70211-6

31. Selby W et al (2007) Two-year combination antibiotic therapy with clarithromycin, rifabutin, and clofazimine for Crohn's disease. Gastroenterology 132:2313-2319. doi:10.1053/j.gastro.2007.03.031

32. Barnich N, Darfeuille-Michaud A (2007) Adherent-invasive Escherichia coli and Crohn's disease. Curr Opin Gastroenterol 23: 16-20. doi:10.1097/MOG.0b013e3280105a38

33. Ohkusa T et al (1993) Bacterial invasion into the colonic mucosa in ulcerative colitis. J Gastroenterol Hepatol 8:116-118

34. Ohkusa T et al (2002) Fusobacterium varium localized in the colonic mucosa of patients with ulcerative colitis stimulates species-specific antibody. J Gastroenterol Hepatol 17:849-853

35. Ohkusa T, Okayasu I, Ogihara T, Morita K, Ogawa M, Sato N (2003) Induction of experimental ulcerative colitis by Fusobacterium varium isolated from colonic mucosa of patients with ulcerative colitis. Gut 52(1):79-83

36. Ohkusa Tet al (2005) Effectiveness of antibiotic combination therapy in patients with active ulcerative colitis: a randomized, controlled pilot trial with long-term follow-up. Scand J Gastroenterol 40:1334 1342. doi: $10.1080 / 00365520510023648$

37. Lees CW et al (2011) New IBD genetics: common pathways with other diseases. Gut 60:1739-1753. doi:10.1136/gut.2009.199679

38. Wehkamp J et al (2004) NOD2 (CARD15) mutations in Crohn's disease are associated with diminished mucosal alpha-defensin expression. Gut 53:1658-1664. doi:10.1136/gut.2003.032805

39. Noguchi E et al (2009) A Crohn's disease-associated NOD2 mutation suppresses transcription of human IL10 by inhibiting activity of the nuclear ribonucleoprotein hnRNP-A1. Nat Immunol 10:471-479. doi:10.1038/ni.1722

40. Cooney R et al (2010) NOD2 stimulation induces autophagy in dendritic cells influencing bacterial handling and antigen presentation. Nat Med 16:90-97. doi:10.1038/nm.2069

41. Salzman NH et al (2010) Enteric defensins are essential regulators of intestinal microbial ecology. Nat Immunol 11:76-83. doi:10.1038/ni. 1825

42. VanDussen KL et al (2014) Genetic variants synthesize to produce paneth cell phenotypes that define subtypes of Crohn's disease. Gastroenterology 146:200-209. doi:10.1053/j.gastro.2013.09.048

43. Cadwell K et al (2010) Virus-plus-susceptibility gene interaction determines Crohn's disease gene Atg16L1 phenotypes in intestine. Cell 141:1135-1145. doi:10.1016/j.cell.2010.05.009
44. Adolph TE et al (2013) Paneth cells as a site of origin for intestinal inflammation. Nature 503:272-276. doi:10.1038/nature12599

45. Kim SC et al (2005) Variable phenotypes of enterocolitis in interleukin 10-deficient mice monoassociated with two different commensal bacteria. Gastroenterology 128:891-906

46. Garrett WS et al (2007) Communicable ulcerative colitis induced by T-bet deficiency in the innate immune system. Cell 131:33-45. doi: 10.1016/j.cell.2007.08.017

47. Qin J et al (2010) A human gut microbial gene catalogue established by metagenomic sequencing. Nature 464:59-65. doi:10.1038/ nature 08821

48. Gill SR et al (2006) Metagenomic analysis of the human distal gut microbiome. Science 312:1355-1359. doi:10.1126/science.1124234

49. Morgan XC et al (2012) Dysfunction of the intestinal microbiome in inflammatory bowel disease and treatment. Genome Biol 13:R79. doi:10.1186/gb-2012-13-9-r79

50. Furusawa $\mathrm{Y}$ et al (2013) Commensal microbe-derived butyrate induces the differentiation of colonic regulatory T cells. Nature 504: 446-450. doi:10.1038/nature 12721

51. Pitcher MC et al (2000) The contribution of sulphate reducing bacteria and 5-aminosalicylic acid to faecal sulphide in patients with ulcerative colitis. Gut 46:64-72

52. Ivanov II et al (2009) Induction of intestinal Th17 cells by segmented filamentous bacteria. Cell 139:485-498. doi:10.1016/j.cell.2009.09.033

53. Caselli $\mathrm{M}$ et al (2013) Segmented filamentous bacteria-like organisms in histological slides of ileo-cecal valves in patients with ulcerative colitis. Am J Gastroenterol 108:860-861. doi:10.1038/ajg. 2013.61

54. van Nood E et al (2013) Duodenal infusion of donor feces for recurrent Clostridium difficile. N Engl J Med 368:407-415. doi:10. 1056/NEJMoa1205037

55. Bennet JD, Brinkman M (1989) Treatment of ulcerative colitis by implantation of normal colonic flora. Lancet 1:164

56. Anderson JL et al (2012) Systematic review: faecal microbiota transplantation in the management of inflammatory bowel disease. Aliment Pharmacol Ther 36:503-516. doi:10.1111/j.1365-2036. 2012.05220.x

57. Angelberger $\mathrm{S}$ et al (2013) Temporal bacterial community dynamics vary among ulcerative colitis patients after fecal microbiota transplantation. Am J Gastroenterol 108:1620-1630. doi:10.1038/ajg.2013.257

58. Kump PK et al (2013) Alteration of intestinal dysbiosis by fecal microbiota transplantation does not induce remission in patients with chronic active ulcerative colitis. Inflamm Bowel Dis 19:2155-2165. doi:10.1097/MIB.0b013e31829ea325

59. Kunde S et al (2013) Safety, tolerability, and clinical response after fecal transplantation in children and young adults with ulcerative colitis. J Pediatr Gastroenterol Nutr 56:597-601. doi:10.1097/MPG. 0b013e318292fa0d

60. Gionchetti P et al (2000) Oral bacteriotherapy as maintenance treatment in patients with chronic pouchitis: a double-blind, placebocontrolled trial. Gastroenterology 119:305-309

61. Mimura T et al (2004) Once daily high dose probiotic therapy (VSL\#3) for maintaining remission in recurrent or refractory pouchitis. Gut 53:108-114

62. Tursi A et al (2010) Treatment of relapsing mild-to-moderate ulcerative colitis with the probiotic VSL\#3 as adjunctive to a standard pharmaceutical treatment: a double-blind, randomized, placebocontrolled study. Am J Gastroenterol 105:2218-2227. doi:10.1038/ ajg. 2010.218

63. Sood A et al (2009) The probiotic preparation, VSL\#3 induces remission in patients with mild-to-moderately active ulcerative colitis. Clin Gastroenterol Hepatol 7:1202-1209. doi:10.1016/j.cgh. 2009.07.016, 1209 e1201

64. Miele E et al (2009) Effect of a probiotic preparation (VSL\#3) on induction and maintenance of remission in children with ulcerative colitis. Am J Gastroenterol 104:437-443. doi:10.1038/ajg.2008.118 
65. Soo I et al (2008) VSL\#3 probiotic upregulates intestinal mucosal alkaline sphingomyelinase and reduces inflammation. Can J Gastroenterol 22:237-242

66. Rembacken BJ et al (1999) Non-pathogenic Escherichia coli versus mesalazine for the treatment of ulcerative colitis: a randomised trial. Lancet 354:635-639

67. Kruis W et al (2004) Maintaining remission of ulcerative colitis with the probiotic Escherichia coli Nissle 1917 is as effective as with standard mesalazine. Gut 53:1617-1623. doi:10.1136/gut.2003.037747

68. Kruis W et al (1997) Double-blind comparison of an oral Escherichia coli preparation and mesalazine in maintaining remission of ulcerative colitis. Aliment Pharmacol Ther 11:853-858

69. Kamada N et al (2008) Nonpathogenic Escherichia coli strain Nissle 1917 inhibits signal transduction in intestinal epithelial cells. Infect Immun 76:214-220. doi:10.1128/iai.01193-07

70. Zocco MA et al (2006) Efficacy of Lactobacillus GG in maintaining remission of ulcerative colitis. Aliment Pharmacol Ther 23:15671574. doi:10.1111/j.1365-2036.2006.02927.x

71. Bousvaros A et al (2005) A randomized, double-blind trial of Lactobacillus GG versus placebo in addition to standard maintenance therapy for children with Crohn's disease. Inflamm Bowel Dis 11: 833-839

72. Schultz $\mathrm{M}$ et al (2004) Lactobacillus GG in inducing and maintaining remission of Crohn's disease. BMC Gastroenterol 4:5. doi:10.1186/ 1471-230x-4-5

73. Furrie E et al (2005) Synbiotic therapy (Bifidobacterium longum/ Synergy 1) initiates resolution of inflammation in patients with active ulcerative colitis: a randomised controlled pilot trial. Gut 54:242249. doi:10.1136/gut.2004.044834

74. Kato K et al (2004) Randomized placebo-controlled trial assessing the effect of bifidobacteria-fermented milk on active ulcerative colitis. Aliment Pharmacol Ther 20:1133-1141. doi:10.1111/j.13652036.2004.02268.x

75. Ishikawa $\mathrm{H}$ et al (2003) Randomized controlled trial of the effect of bifidobacteria-fermented milk on ulcerative colitis. J Am Coll Nutr 22:56-63

76. Hayashi A et al (2013) A single strain of Clostridium butyricum induces intestinal IL-10-producing macrophages to suppress acute experimental colitis in mice. Cell Host Microbe 13:711-722. doi:10.1016/j.chom.2013.05.013 